\title{
Bouvard et Pécuchet, le roman impossible des savoirs
}

\section{Pierre Campion}

\section{(2) OpenEdition \\ Journals}

Édition électronique

URL : http://journals.openedition.org/aes/546

DOI : 10.4000/aes.546

ISSN : 2258-093X

Éditeur

Laboratoire LISAA

Référence électronique

Pierre Campion, «Bouvard et Pécuchet, le roman impossible des savoirs », Arts et Savoirs [En ligne],

1 | 2012, mis en ligne le 15 février 2012, consulté le 30 avril 2019. URL : http://

journals.openedition.org/aes/546; DOI : 10.4000/aes.546

Ce document a été généré automatiquement le 30 avril 2019

Centre de recherche LISAA (Littératures SAvoirs et Arts) 


\title{
Bouvard et Pécuchet, le roman impossible des savoirs
}

\author{
Pierre Campion
}

1 Dans les jours où Flaubert allait se mettre à rédiger Bouvard et Pécuchet, Tourgueniev exprimait des réserves sur le projet dont son ami lui avait parlé : « C'est un sujet à traiter presto, à la Swift, à la Voltaire. [...] Si vous vous appesantissez là-dessus, si vous êtes trop savant... »'. Glissez, mortels! Flaubert lui répond fermement, mais non sans révéler ses doutes et ses craintes :

\begin{abstract}
Il n'y a plus à reculer. Mais quelle peur j'éprouve ! Quelles transes ! Il me semble que je vais m'embarquer pour un très grand voyage vers des régions inconnues, et que je n'en reviendrai pas.

Malgré l'immense respect que j'ai pour votre sens critique [...] je ne suis point de votre avis sur la manière dont il faut prendre ce sujet-là. S'il est traité brièvement, d'une façon concise et légère, ce sera une fantaisie plus ou [moins] spirituelle, mais sans portée et sans vraisemblance, tandis qu'en détaillant et en développant, j'aurai l'air de croire à mon histoire, et on peut faire une chose sérieuse et même effrayante. Le grand danger est la monotonie et l'ennui. Voilà bien ce qui m'effraie cependant... [...] Je ne puis exposer une idée sans aller jusqu'au bout. ${ }^{2}$
\end{abstract}

2 En connaissance de cause, Flaubert a choisi entre deux perspectives possibles. Il y avait bien dans le sujet la matière d'un Candide, et on retrouvera en effet quelque chose de cela dans les deux bonshommes, ainsi que, si l'on y regarde bien, l'idée de courtes scènes et la cruauté de l'ironie. Mais la décision a été pesée et elle est définitive : ce sera une longue histoire, complète et par là convaincante, pesant du poids des choses, au risque de l'ennui. L'engagement est lucide, l'exécution commencera dans quelques jours, ce sera une aventure, il ira jusqu'au bout. On n'attendait pas forcément ce ton d'héroïsme.

Pour passer outre aux conseils avisés d'un confrère et ami, et à ses propres appréhensions, et pour persister pendant six années dans ce projet dont l'auteur luimême voit d'avance les difficultés inquiétantes, dont il éprouvera sans cesse les dégoûts et dont, de fait, il ne verra pas la fin, il faut de bien sérieuses raisons. D'abord, et de manière assez étrange, l'écrivain invoque la nécessité de conférer une crédibilité à son histoire, comme si elle en manquait à ses propres yeux, et de lui donner le tour d'« une 
chose sérieuse et même effrayante ", comme si le thème du savoir mettait en jeu des significations et des puissances redoutables. Bien sûr, Bouvard et Pécuchet ne seront pas des Faust ou des Balthazar Claës, mais la dimension des enjeux exclut, aux yeux de Flaubert, tout ce qui serait allusion, fantaisie, irresponsabilité. Et puis il y a une disposition intime et en somme assez peu voltairienne, que la Correspondance évoquera souvent pendant les années de l'écriture, celle de cracher brutalement le dégoût de l'époque, de vomir le venin qui l'étouffe ${ }^{3}$. Enfin il y a la vocation d'un écrivain qui est, éthique ambivalente et esthétique ambiguë, de dénoncer et d'exalter en même temps l'inhumanité écœurante de la réalité, dans l'homme et dans les choses, - une inhumanité appelée, dans toute son œuvre, la bêtise, haïssable et fascinante. Voltaire s'en dégage d'un saut, Flaubert ne craint pas de s'y enfoncer.

\section{Au bout de l'idée}

4 En littérature, comme ailleurs, il est des idées qu'il suffit d'indiquer ou de suggérer par un seul trait, quitte à reprendre celui-ci à neuf, sous une tout autre formulation : « Il y a du mal sur la terre", "Aimez ce que jamais on ne verra deux fois ", «Un peu profond ruisseau calomnié la mort »... Il en est d'autres qui ne tiennent que par la force démonstrative de leur développement.

5 L'histoire d'Emma Bovary et celle de Frédéric Moreau relataient le long affrontement de deux âmes à la réalité des êtres, des choses et des événements, à leur propre médiocrité aussi, laquelle n'était que l'une des formes, intérieure et ennemie, immuable, de cette réalité. Chacun des deux grands récits, différemment bien sûr, s'efforçait de dresser, au regard de cette réalité, l'exigence de ces deux personnages et, à travers l'expression de cette exigence, la protestation insistante d'une écriture. La grandeur de Flaubert, c'est de construire - d'essayer de constituer - des romans (des « livres sur rien») qui aient par eux-mêmes l'évidence et la continuité, la force et la nécessité, la crédibilité de ce à quoi, tout uniment, il se suffit d'être. Ainsi l'histoire de Madame Bovary revêt-elle la cohérence impitoyable obtenue au prix des tourments que l'on sait et celle de L'Éducation sentimentale cette espèce de flux de la vie tel qu'il laisse de côté un jeune homme avide de s'y plonger et finalement désespéré de ne pas pouvoir le faire ni même d'en avoir eu vraiment l'envie. Dans ces deux œuvres, et aussi dans sa Tentation de Saint Antoine, Flaubert allait jusqu'au bout de son idée, parce que celle-ci devait transposer au sein de son propre développement l'implacable continuité, aussi massive et détaillée que possible, qu'il voulait opposer à celle de la réalité. Dans les trois romans, l'ironie était là pour signifier, ostinato, à travers les deux ordres de voix qu'elle met en jeu - celles des personnages et celle de la narration - à la fois la vanité de l'imagination et la légitimité de son exigence : rien ne peut faire que la réalité soit conforme à notre désir mais aussi rien ne peut faire que, en présence de la réalité, l'imagination cède quoi que ce soit de ses droits. Cela au risque, plus présent que jamais dans Bouvard et Pécuchet, de s'engager, par une sorte de perversion, dans l'univers de la Bêtise et de s'y perdre, à tenter de le singer, de le concurrencer et le défier, de le vaincre, dans l'ordre où il se déploie, le temps.

En 1874, après les aventures de l'imagination, et leur espèce de grandeur, Flaubert entend écrire les longues mésaventures du savoir, dans sa marche, qui devrait être celle d'un affranchissement. À leur manière, dévoyée et pitoyable, de fait les deux bonshommes entreprennent de suivre la devise des Lumières, qu'ils ont pu rencontrer dans leurs lectures, et telle qu'elle a été formulée par Kant: "Accéder aux Lumières consiste pour 
l'homme à sortir de la minorité où il se trouve par sa propre faute. [...] Sapere aude! Aie le courage de te servir de ton propre entendement. $»^{4}$ De l'audace ils en ont, ou plutôt de la témérité, celle des autodidactes, non informée, irréfléchie, et tout de suite déçue, rabrouée et bafouée. Quant à la sagesse (sapientia), ils ne sauront jamais ce que c'est : ils connaissent et éprouvent l'obligation de l'émancipation, pour eux-mêmes et pour les autres, mais ils resteront des enfants, plus naifs finalement que Victor et Victorine, et voués à la punition de la copie, c'est-à-dire, mais en pire, à un état qu'ils n'auraient jamais dû quitter. À moins que cela justement ce ne soit la sagesse, élémentaire mais durement acquise, de reconnaittre leur condition, humaine, et d'y retourner non pas sciemment mais au moins délibérément...

\section{En présence de la réalité}

7 Qu'est-ce que la réalité ? C'est l'espèce de fin de non-recevoir que les choses, les êtres et les événements opposent à notre demande de sens, d'amour et de simple existence, par le seul fait que les choses et les êtres sont et que les événements adviennent.

8 Cette fin de non-recevoir, chacun des deux bonshommes l'éprouvait sans doute dans son existence étriquée, sans trop le savoir et d'une manière en somme bénigne. Dans la ville déserte et brûlante, leur rencontre leur révèle qu'ils sont deux à en souffrir, et cela change tout. Presque aussitôt, l'héritage de Bouvard va libérer leur rêve d'autonomie et de vie heureuse parmi les choses. S'acheter une maison, vivre à la campagne, activer leurs énergies dans un monde selon leur cœur :

Déjà, ils se voyaient en manches de chemise, au bord d'une plate-bande émondant des rosiers, et bêchant, binant, maniant de la terre, dépotant des tulipes. [...] Plus d'écritures! plus de chefs! plus même de terme à payer ! - Car ils posséderaient un domicile à eux ! Et ils mangeraient les poules de leur basse-cour, les légumes de leur jardin, et dîneraient en gardant leurs sabots! - «Nous ferons tout ce qui nous plaira! Nous laisserons pousser notre barbe ! $»^{5}$

On ne parle pas encore vraiment des livres : « Bouvard tranchait la question [de "savoir si tel livre était vraiment un livre de bibliothèque"]: "Eh! nous n'aurons pas besoin de bibliothèque." - "D'ailleurs, j'ai la mienne" disait Pécuchet " ${ }^{6}$. C'est l'échec de leurs cultures et conserves qui les y conduira. Bientôt, échouant en tout, ils ne pourront plus rien faire sans les livres.

Cependant, dès l'abord, la campagne où ils vont se retirer se révèle ingrate :

[...] la route, toujours la même, s'allongeait en montant jusqu'au bord de l'horizon. Les mètres de cailloux se succédaient, les fossés étaient pleins d'eau, la campagne s'étalait par grandes surfaces d'un vert monotone et froid, des nuages couraient dans le ciel, de temps à autre la pluie tombait ${ }^{7}$.

Un paysage sans grâce, pour ainsi dire activement et inconsciemment indifférent, saturé de cette indifférence. C'est ainsi. Cette fin de non-recevoir, ils l'éprouveront aussi dans l'ingratitude de Petit et de Gorju ou des enfants qu'ils ont prétendu aimer et sauver. Ils l'éprouvent encore dans les accidents qui arrêtent leurs entreprises, même quand leur maladresse ou leur incurie n'y est pour rien. Michel Foucault écrit quelque part : «De Bouvard et Pécuchet peut-on dire qu'ils se trompent? qu'ils commettent des erreurs dès que la moindre occasion leur en est fournie ? [...] L'échec leur vient de toute façon, quoi qu'ils fassent, qu'ils aient ou non appliqué les règles, que le livre consulté ait été bon ou mauvais. À leur entreprise, n'importe quoi arrive, l'erreur bien sûr, mais l'incendie, le gel, 
la sottise et la méchanceté des hommes, la colère d'un chien. [...] Dans les catégories, on erre; hors d'elles, au-dessus d'elles, on est bête. Bouvard et Pécuchet sont des êtres acatégoriques. $»^{8}$

Désespérément, à force de savoirs, ils essaieront de faire entrer le réel dans les catégories de la pensée et dans la familiarité des humains, et ils y échoueront. Alors, dernier effort de leur philosophie, ils tentent le suicide :

Quelque chose d'irrévocable était venu. [...] N'importe quoi valait mieux que cette existence monotone, absurde, et sans espoir. [...] L'ambition de Pécuchet était le savoir philosophique. Or, le plus vaste des problèmes, celui qui contient tous les autres, peut se résoudre en une minute. Quand donc arriverait-elle?

- «Autant tout de suite, en finir.»

- « Comme tu voudras » dit Bouvard.

Et ils examinèrent la question du suicide. ${ }^{9}$

Une fois de plus, ils ne vont pas au bout de leur idée. La nuit de Noël, ayant tenté les extrémités de la philosophie et de la tragédie, ivres l'un d'alcool et l'autre de faim, et parce qu'ils n'ont pas fait leur testament - philosophique? -, ils abandonnent. Ils tombent en religion. Ils vont s'abîmer définitivement dans la bêtise des croyances et des savoirs :

Pas de réflexion! Copions! Il faut que la page s'emplisse, que "le monument » se complète. - Égalité de tout, du bien et du mal, du beau et du laid, de l'insignifiant et du caractéristique. Il n'y a pas de vrai que les phénomènes. -

Finir par la vue des deux bonshommes penchés sur leur pupitre, et copiant. ${ }^{10}$ Voilà, à peu près, ce que devait nous dire la fin du roman, si celui-ci avait trouvé sa fin.

\section{Le savoir et les savoirs}

Le sens de la réalité n'est pas un savoir. Lui aussi, il est hors des catégories du savoir : il ne s'apprend pas, et surtout pas dans les livres. Il est une forme de la foi, de la seule qui échappe à toute illusion, à toute bigoterie, la seule qui fasse entrer la pensée dans une bêtise supérieure, celle, peut-être, qui fait réintégrer saint Antoine dans l'indistinction de la Matière. Croire à ce qui est, à ce qui ne se démontre ni se déduit, à ce qui, aussi bien, s'oublie au fil des vies ou se perd et même, dans certaines, n'aura jamais été reconnu : à la réalité du réel. Mais voilà, les deux bonshommes sont exactement dans l'ordre opposé à celui du sens de la réalité, ils se maintiennent dans l'ordre où se loge une bêtise noire et inconsciente d'elle-même, une foi encore mais perverse, une croyance têtue, rétive à toutes les épreuves : croire à ce qui n'est pas ${ }^{11}$.

Qu'est-ce que le savoir, en chacun des savoirs, mais aussi en tant que tel ? Comme l'amour ou le pouvoir, mais bien spécifiquement, c'est l'une des formes, celle-ci fondamentale peut-être et en tout cas dernière dans l'œuvre de Flaubert, selon lesquelles nous tentons de nous approprier le réel. Ce ne peut être qu'une illusion, des plus pernicieuses. Car on ne saurait s'approprier le réel : le réel est ce qui est au-delà ou en deçà des catégories de la pensée. Comme toutes les autres, cette illusion-là est native - naïve - et, comme telle, tenace et même invincible. Elle suppose que la réalité relève de nos catégories de pensée, lesquelles ne seraient pas seulement des conditions du savoir mais aussi celles de la communauté humaine. L'illusion du savoir est celle de nos appartenances, et la critique du savoir les visera donc toutes ${ }^{12}$.

Seulement, on ne saurait atteindre ni dénoncer le Savoir en soi, lequel en effet n'est qu'une forme première et invétérée de cette illusion-là, la croyance en une espèce 
d'immanence de la pensée - de toutes les pensées, raisonnées ou sauvages - au monde, aux autres et à nous-mêmes : la masse accumulée et indistincte de concrétions mortes ou vivantes, le for souverain des lieux communs et des idées reçues. Aussitôt il faut donc détailler le savoir en savoirs que l'on pourra isoler, fût-ce en articles de dictionnaire, raconter chacun et le déjouer. Car alors on pourra faire l'histoire de chaque savoir et, par le récit de sa constitution puis de sa déception, le démonter. Raconter tel et tel savoir, non pas selon une histoire des sciences mais dans le processus réel de sa constitution, c'est-à-dire dans la vie de tel personnage. Le romancier ne peut pas évoquer directement le Savoir, ni même les savoirs, il lui faut passer par la mise en scène de la relation humaine aux savoirs, par une sorte de phénoménologie romanesque, dont il y avait une première esquisse avec Homais et une réalisation, très différente par les modalités et le sens, dans saint Antoine.

Alors on pourra attaquer de l'intérieur ce processus-là, c'est-à-dire monter un dispositif d'ironie qui en désarme et dissolve la nocivité telle qu'elle est à l'œuvre dans la conscience de ce personnage. Ironiser, c'est empiéter sur la forteresse vide du savoir : un pied dedans, un pied dehors, pour peser, disjoindre, ouvrir. Ainsi, dans telle phrase (et, à la limite, dans tout le roman): "Mais bientôt ils s'ennuyèrent, leur esprit ayant besoin d'un travail, leur existence d'un but! $»^{13}$ Qui s'exclame ici ? La parole intime de chacun des deux bonshommes, ou celle qu'ils échangent entre eux, ou celle de la narration? Toutes celles-là, bien sûr...

18 Car le savoir est une illusion, et l'illusion n'est pas une erreur que l'on puisse réfuter par ordre et arguments, c'est une passion à déraciner, un état de dépendance qui met Bouvard et Pécuchet hors des catégories du discours rationnel et de la Raison même. Il faut entrer dans les errances de leur pensée, dans leur détail et dans leur mouvement, c'est-à-dire dans leur ordre de réalité : constituer une histoire de cette réalité aussi convaincante que l'est toute réalité, une histoire à laquelle on croie, et l'auteur tout le premier.

\section{Faire le tour des savoirs}

Cette décision prise, il faudra faire l'encyclopédie des savoirs. Car tant que l'on n'a pas critiqué singulièrement tous les savoirs, l'illusion que l'un d'entre eux serait fondé persistera dans le sujet et la notion du savoir y demeurera intacte. Prendre un par un les savoirs, c'est pénétrer dans leur cercle, les spécifier, les mettre en contradiction chacun avec lui-même et avec tous les autres, briser l'enchantement propre du Savoir qui est de prétendre totaliser les pouvoirs d'appropriation supposés des hommes à l'égard des choses et à l'égard d'eux-mêmes. Dans le même mouvement, on pourra démembrer la réalité elle-même, s'immiscer dans le mur sans faille qu'elle nous oppose par elle-même et surtout à travers notre croyance selon laquelle cette totalité infrangible peut nous être cependant assimilable. Loin d'élever à son tour le mur illusoire d'une nouvelle narration (l'erreur de Madame Bovary?), le roman devra donc être "l'histoire de ces deux bonshommes qui copient, une espèce d'encyclopédie critique en farce $»^{14}$ ou encore « une espèce d'encyclopédie de la Bêtise moderne ${ }^{15}$, l'histoire encyclopédique réussie d'une tentative encyclopédique, et de son échec. Qui ne voit l'ambiguïté et l'ambivalence d'un tel projet? par l'ordre raisonné que celui-ci mettait dans les savoirs, puis selon l'ordre alphabétique 
qu'elle instituait entre les articles : ainsi les savoirs étaient-ils exposés un par un à la Raison, tout en se référant les uns aux autres par un système de renvois, sous la garantie arbitraire et faussement neutre - de l'ordre des mots dans la langue. Ici, il y aura bien un dictionnaire, mais ce sera celui des idées reçues, et surtout il y aura une histoire romanesque, celle de l'odyssée de deux imbéciles dans l'océan des savoirs. Un siècle plus tard, Michel Foucault, lui, fera une archéologie des savoirs : à cet immense fatras, où Flaubert ne veut voir que les contradictions et les délires de la raison, il conférera le genre de nécessité que revêtent, selon lui et par principe, toutes les productions humaines de sens. Comme celle de l'anthropologie structurale, la critique par l'archéologie n'est pas dans la dérision, elle examine les conditions de possibilité et la diversification du savoir : il y aurait de l'ordre dans le sol confus de nos savoirs, à la critique de le retrouver et de le construire.

21 Selon son point de vue à lui, l'auteur de L'Archéologie du savoir et Les Mots et les choses rencontrera donc Bouvard et Pécuchet et il y décèlera la critique d'un encyclopédisme bien particulier, celui dans lequel une volonté de pratiquer le réel débouche sur une folie de connaissances. Déployant le lien originaire que ce livre entretient avec le Saint Antoine, il note les similitudes: "Mêmes éléments : un livre fait de livres; l'encyclopédie érudite d'une culture ; la tentation au milieu de la retraite ; la longue suite des épreuves ; les jeux de la chimère et de la croyance. ${ }^{16}$ Mais il décèle les différences, substantielles : là où le saint s'enferme dans la hantise d'un Livre imaginaire fait des phrases arrachées indifféremment à tous les livres, les deux bonshommes, "pèlerins que rien ne fatigue ", s'activent à parcourir la bibliothèque des livres réels, au besoin de leurs expérimentations, agricoles ou autres, que leur petite fortune les met d'un coup en mesure de réaliser : « Ils essaient tout, s'approchent de tout, touchent à tout ; ils mettent tout à l'épreuve de leur petite industrie. S'ils ont fait retraite, comme le moine d'Égypte, c'est une retraite active, une oisiveté entreprenante dans laquelle ils convoquent, à grand renfort de lectures, tout le sérieux de la science, avec les vérités les plus gravement imprimées. Ce qu'ils ont lu, ils veulent le faire [...]. . ${ }^{17}$

Le sérieux supposé de la science, mais non la démarche de la science, raisonnée et soumise à l'expérience, formée, non immédiatement utilitaire : car ils prétendent, au hasard des occasions et sans méthode, faire de la recherche (géologie, paléontologie, archéologie, histoire), expérimenter (en zoologie et en médecine), inventer des produits (la bouvarine)... Les deux bonshommes en ont indistinctement à tout ce qui se présente : aux choses (à connaître et à transformer), aux êtres (à soigner ou à instruire), aux savoirs (comme connaissances d'emblée disponibles, adéquates et simples), aux livres, comme médiateurs des savoirs (issus de ces savoirs et destinés à y retourner pour les grossir). Faire le tour des livres pour posséder la réalité par l'action et par la pensée réunis : ils sont au-delà de l'incompétence et de la compétence, de l'erreur ou de la vérité, du beau et du laid. Ils ne sont pas en butte à leurs seules insuffisances ou à de malheureux hasards, mais à la réalité.

Dans Flaubert, le principe de ce voyage encyclopédique est ironique, puisque la narration met en perspective le point de vue de ceux qui croient pouvoir parcourir le cercle enchanté. Mais il est périlleux parce que, pour ce faire, le roman lui-même doit se faire encyclopédie, entrer dans toutes les aventures de l'illusion, cela au risque mortel de l'ennui ${ }^{18}$. Périlleux aussi parce qu'il doit faire croire à sa propre réalité, détaillée et développée, au risque d'élever à nouveau, quoique sous une autre forme, un mur de narration. Dès le début, cette entreprise était un défi. Flaubert était prévenu, il le savait 
mieux que personne et la suite du travail ne sera qu'une alternance de désespoirs et d'exaltations, dont celle-ci donne une idée, deux mois à peine après le début de la rédaction :

Au mois d'août, je me suis mis à mon bouquin dont les premières pages m'ont semblé impossibles. J'ai cru un moment que je [ne] pourrais pas continuer! et ma désolation était indescriptible! mais enfin, j'y suis, ça va! (en attendant quelque désespoir nouveau). Si je mène à bien une pareille œuvre, la terre ne sera pas digne de me porter! mais j'ai peur de faire un four absolu. C'est abominable d'exécution! et puis je suis seul maintenant. Absolument seul, sans conseil, ni sans encouragement, pas le moindre secours! rien! La terre me semble vide de tout esprit. La Bêtise universelle s'étale. ${ }^{19}$

Seul comme Gustave Flaubert, qui se donne à constituer solidement le roman de ce vide, à écrire la prose essentiellement ironique de ce monde sans style, une sorte de mission impossible, devant laquelle « il n'y a plus à reculer ».

C'est là que réside l'idée de Bouvard et Pécuchet, laquelle doit être menée à son terme : pour en venir à bout, il faut faire le tour des savoirs, le presto est impossible. Tâche épuisante et probablement infinie, car il n'existe pas de liste exhaustive des savoirs, ne serait-ce que parce que chacun est en prolifération incessante et que cette liste même est désormais en mouvement ${ }^{20}$. Certes, il n'est pas sûr que Flaubert ait succombé à cette tâche, mais il est mort avant de l'avoir achevée : littéralement, il n'est pas revenu de ce grand voyage circulaire.

\section{Bouvard et Pécuchet, l'un et l'autre}

Dès l'origine encore, une autre décision, de romancier, une intuition fondamentale, qui est aussi une précaution. Le candide se divise, il se détaille : les copistes, ou cloportes, ou bonshommes, sont deux. Candide était seul parce qu'il figure dans une féerie et qu'il est flanqué des autres marionnettes que sont Pangloss, Martin ou Cunégonde. Quant à l'Autodidacte de Sartre, il appartient à une histoire, d'ailleurs fortement dramatisée, qui n'est pas la sienne.

Non, si le sujet des savoirs se divise d'emblée entre Bouvard et Pécuchet, c'est parce que le récit de cette odyssée nécessite une scission dans ce sujet, qui lui fasse perdre son caractère abstrait d'absolu non maniable. Car comment insinuer un mouvement romanesque dans l'encyclopédie des savoirs sans supposer une différenciation dans ce sujet, laquelle en effet fasse apparaître et inscrive dans la réalité, à travers une figure double et selon son évolution, les effets clivants des savoirs, dans cette figure même et dans ses relations aux autres? Dans une petite communauté campagnarde, il y aura ceux qui savent plus ou moins et ceux qui prétendent savoir et, entre les deux personnes qui pensent savoir, des possibilités d'entraide, des différences d'intérêts et de points de vue, des discussions et des conflits, des relations spécifiques avec le bloc détaillé des nonsavants, bref les incidents et les événements d'une histoire. Ainsi cette différenciation crée-t-elle deux caractères, lesquels doivent échanger entre eux les effets de réalité : l'Autodidacte serait ici une figure abstraite et mortelle. Leur dualité les concrétise entre grand maigre et petit gros, pauvre et riche, expansif et réservé, crédule et sceptique, forte tête et tête folle, prude et gaillard, etc. Mais cette différenciation ne se fait pas sans schématisme, et, à chaque épreuve et malgré les efforts de Flaubert, on peine un peu à identifier à nouveau chaque protagoniste, tellement le système de l'espoir et de la 
déception tend à raboter la personne de chacun au profit de la commune mésaventure ${ }^{21}$. Retenant à sa manière l'idée d'Aristote et voulant oublier peut-être que l'histoire détermine les caractères et non l'inverse, Flaubert cherche à sauver son histoire en créant d'emblée deux personnages.

\section{Dramatiser ?} La première date explicitement indiquée est le 20 janvier 1839, le jour où Bouvard apprend son héritage ; à travers une allusion à l'expédition italienne de Napoléon III, la dernière date évoquée sera 1859 (ch. VI, p. 344, une histoire qui dure déjà depuis plus de vingt ans...). Vers la fin de 1840, la ferme est payée et, le 20 mars (1841 ?), ils déménagent pour leur campagne. Dans l'été 1845, ils lisent des livres d'histoire de France. Et, « dans la matinée du 25 février 1848, on apprit à Chavignolles, par un individu venu de Falaise, que Paris était couvert de barricades - et le lendemain, la proclamation de la République fut affichée sur la mairie. Ce grand événement stupéfia les bourgeois » (ch. VI, début). Juste avant, à la fin du chapitre V : « - "Il me semble" dit Pécuchet "que nous aurons bientôt du grabuge ?" Car il voyait tout en noir, peut-être à cause de sa jaunisse. » Leur vie entre alors dans les événements de 1848-1851, tels qu'ils se répercutent à Chavignolles. Ces événements raniment d'abord leur zèle libéral et relancent leurs illusions, puis les isolent peu à peu et même les mettent en danger auprès des autorités. Plus que jamais traitée en farce, l'irruption de l'Histoire dans leur histoire n'aura fait que cristalliser leurs obsessions et radicaliser leurs échecs.

Ordonner en moments significatifs l'improvisation des actions et le fatras des savoirs, et relier ces moments par des événements et de la causalité - celle-ci fût-elle absurde ou cacophonique -, voilà comment Flaubert construit son roman. Il y aura donc des périodes, organisées selon des thèmes et disposées en chapitres: ceux de l'agriculture (II), de l'histoire naturelle (III), de l'archéologie (IV), de l'histoire (V), de la politique (VI), de la dépression nerveuse (VII), des sciences de l'homme (VII, gymnastique, occultisme, philosophie...), de la religion (VIII), de l'éducation (X). Le chapitre final de la conférence devait prolonger le précédent en détaillant aussi les déboires accumulés des deux héros auprès de leurs concitoyens et des autorités. De l'un à l'autre, on passe par des transitions soigneusement signalées. L'accident de l'alambic les mène à l'étude de la chimie, le bahut de Gorju à l'archéologie, leur dépression les ramène à la religion, mais pour une conversion qui n'a rien de définitif. Et ainsi de suite.

31

autre part, le romancier crée un petit monde de personnages secondaires et ménage des intrigues qui traversent le roman : les amours de Bouvard et de Mme Bordin empêchées par les manœuvres de celle-ci pour acheter la ferme, les aventures et les amours de Gorju (et leurs incidences sur la vie intime de Pécuchet), l'histoire de Mélie et de Pécuchet, celle de Victor et Victorine, l'hostilité grandissante des notables et de la gendarmerie... Ces intrigues prennent le relais l'une de l'autre pour soutenir l'intérêt et le relancer même. 

lassitude et la fatigue (toute une physiologie des savoirs), la saturation, l'ennui, les problèmes d'argent, l'infortune des choses - l'échec sous toutes ses formes -, au péril évident pour le roman de la monotonie et de la perte de l'intérêt. Délibérément, Flaubert avait renoncé au mouvement unitaire qui animait Madame Bovary. Emma aussi allait d'échec en échec, mais suivant la nécessité passionnée et passionnante d'un destin.

\section{L'illusion et la désillusion}

$\mathrm{Au}$ théâtre et dans le roman, de la tragédie grecque aux Mots de Sartre, la désillusion est un processus puissant et mystérieux, puissant esthétiquement parce que non insérable dans les raisons de la durée, et indécomposable à l'analyse narrative. Dans Les Mots, à la fin, Sartre constate la désillusion : il ne croit plus à la littérature. Toutefois il reporte à une autre fois le récit de la désillusion elle-même: «J'ai changé. Je raconterai plus tard quels acides ont rongé les transparences déformantes qui m'enveloppaient, quand et comment j'ai fait l'apprentissage de la violence, découvert ma laideur [...]. Je vois clair, je suis désabusé, je connais mes vraies tâches [...]; depuis à peu près dix ans je suis un homme qui s'éveille, guéri d'une longue, amère et douce folie et qui n'en revient pas et qui ne peut se rappeler sans rire ses anciens errements et qui ne sait plus que faire de sa $v^{2} e^{22}$. À cause peut-être de la cécité qui a saisi l'écrivain, ou par une impossibilité d'un autre ordre, littéraire ou philosophique, ce récit n'est pas venu. Reste le constat d'une sortie hors de la littérature, dans une œuvre où partout se reconnaît aisément la prégnance de la grande littérature classique...

Selon la poétique du dramatique, la désillusion est un événement qui, renversant le processus de l'illusion, inaugure le temps immuable et le règne de la Vérité. Dans le mouvement qui va de l'illusion à la désillusion, le principe et l'intérêt de la péripétie c'est qu'une logique malheureuse et fondée sur la puissance même du temps puisse, contre l'attente, être arrêtée, déviée et même retournée, et que cela s'accomplisse, même dans l'horreur, pour le bien de la vérité. Or, dans Bouvard et Pécuchet, le processus de la désillusion est répétitif et interminable, et c'est comme si le principe fondateur du genre romanesque en était perverti. La seule vérité qui éclate, et cela à chaque moment du roman, c'est que l'illusion revient toujours, c'est-à-dire qu'il n'y a pas de désillusion. Ici, l'illusion n'est pas le moment malheureux d'une histoire mais l'état irrévocable de la vie humaine, constitué depuis toujours et pour toujours, y compris et surtout quand les humains prétendent à la vérité que procureraient le savoir et la philosophie, - la philosophie, un savoir comme un autre. Car le projet précisément, dans Bouvard et Pécuchet, mais de manière non dramatique, ce n'est pas seulement de faire la vérité sur des caractères, des événements, des destins, mais sur le besoin immuable, sur l'exigence et sur l'impossibilité de la véritéén.

Pas de dénouement, puisqu'il n'y a pas eu de nœud. Au début, on a une rencontre de hasard que les deux protagonistes qualifieront de providentielle : deux hommes marchant l'un vers l'autre sur un boulevard désert, deux chapeaux marqués de la même manière, une chambre surchauffée, celle de Pécuchet, encombrée de livres et de poussière, un bavardage, une altercation avec un garçon de café, puis un héritage inattendu... Ces circonstances permettent à deux êtres quelconques de découvrir chacun dans l'autre le miroir de son existence malheureuse, de laquelle il croit pouvoir sortir par la reconnaissance d'une âme sœur en matière d'insatisfaction et de vagues désirs. 
Maintenant qu'ils sont deux, ils croient déjà qu'ils vont avoir une destinée. À la fin, ils retournent à la copie : « Ils copièrent... tout ce qui leur tomba sous la main... [...] Finir par la vue des deux bonshommes penchés sur leur pupitre, et copiant.» De la copie comme métier à la copie de tout ce qui a été écrit, il n'y aura eu lieu que les répétitions de l'illusion. Rien ne dit qu'ils ne croient plus aux savoirs, au contraire. Mais c'est d'une croyance étrange et pure, vidée de contenu et de sens : Bouvard et Pécuchet ne servent plus qu'à "remplir la page ", à faire que "le monument " se complète, à recycler le mur des mots - « vieux papiers achetés au poids à la manufacture de papiers voisine »-, que les humains s'affairent depuis toujours à élever contre la réalité du réel. Obéir à un commandement que personne n'a édicté... Quelque chose ici, dans les situations, le ton, le style, sonne d'avance comme un air qui, un jour, nous sera familier : «Seul comme Franz Kafka. » Emma attirait la foudre; eux ne sont que deux êtres ordinaires qui auraient pu, comme beaucoup de gens, passer à côté de la réalité, sans histoires et sans même le savoir.

La leçon de Bouvard et Pécuchet : la seule chose que l'on sache de l'illusion, c'est qu'elle est invincible. Le seul récit que l'on puisse en faire, c'est celui de ses infinis recommencements. Le seul intérêt que l'on puisse prendre à cette fable, c'est à la diversité de ses variations, que la dernière couronne brillamment en somme : la narration n'aura fait sans doute que mettre du mouvement dans l'immobilité de l'Illusion.

On sait que, à l'époque de Bouvard et Pécuchet, Flaubert découvrait et lisait Schopenhauer ${ }^{24}$ . Il ne faut pas penser à une influence mais plutôt à une rencontre entre un écrivain féru de style et un philosophe peu soucieux d'écriture, que réunissent un mépris des savoirs et de l'amour -, un dégoût pour toutes les illusions et leurs ruses infinies, une obsession pour les répétitions, une passion sans espoir pour la réalité.

\section{NOTES}

1. Tourgueniev à Flaubert, 12 juillet 1874, dans Gustave Flaubert, Correspondance [Corr.], Gallimard, coll. «Bibliothèque de la Pléiade », IV, p. 833.

2. Flaubert à Tourgueniev, 29 juillet 1874, Corr., IV, p. 843.

3. Voir notamment la lettre de Flaubert à Ernest Feydeau, 28 octobre 1872: "Mais avant de crever, ou plutôt en attendant une crevaison, je désire "vuider" le fiel dont je suis plein. Donc, je prépare mon vomissement. Il sera copieux et amer, je t'en réponds. » (Corr., IV, p. 596).

4. Kant, Qu'est-ce que les Lumières? (1784), présentation et trad. de J.-M. Muglioni, Hatier, 1999, p. 4.

5. Bouvard et Pécuchet, présentation par Stéphanie Dord-Crouslé, Flammarion, coll. « GF », 1999, I, p. 59. Nous citerons le texte dans cette édition.

6. Id., p. 60.

7. Id., p. 62-63.

8. Michel Foucault, «Theatrum philosophicum », dans Critique, novembre 1970, repris dans Dits et écrits I. 1954-1975, Gallimard, coll. « Quarto », 1994-2001, p. 960.

9. Bouvard et Pécuchet, VIII, op. cit., p. 306-307.

10. Bouvard et Pécuchet, «Conclusion », op. cit., p. 401. 
11. Michel Foucault, à propos de Bouvard et Pécuchet: «Si grande est la pureté de leur entreprise que leur échec, s'il leur montre l'incertitude de telle proposition ou de telle science, n'entame jamais la solidité de leur croyance au savoir en général. Les désastres restent extérieurs à la souveraineté de leur foi : celle-ci demeure intacte. [...] Ils sont l'image de Job dans le monde moderne : atteints moins dans leurs biens que dans leur savoir, abandonnés non de Dieu mais de la Science, ils maintiennent comme lui leur fidélité ; ce sont des saints " («La Bibliothèque fantastique ", dans Travail de Flaubert, présentation de Gérard Genette, Seuil, coll. "PointsLittérature ", 1983, p. 119). Ce texte est paru d'abord en 1964, sous la forme d'une préface sans titre à une édition allemande de La Tentation de Saint Antoine, puis en 1970 et enfin dans le recueil indiqué ci-dessus. Voir la première version dans Michel Foucault, Dits et écrits I, op. cit., p. 321-353. 12. Outre le «Dictionnaire des idées reçues", le dossier de Bouvard et Pécuchet comporte des notes sur l'esthétique. Raison théorique, raison pratique, jugement de goût, tout l'édifice kantien y passe.

13. Bouvard et Pécuchet, X, op. cit., p. 393.

14. Flaubert à Edma Roger des Genettes, 19 août 1872, Corr., IV, p. 559. Notons la date, antérieure de deux ans au début de la rédaction.

15. Flaubert à Adèle Perrot, 17 octobre 1872, Corr., IV, p. 590.

16. Michel Foucault, « La Bibliothèque fantastique », op. cit., p. 118.

17. Ibid., p. 119.

18. Flaubert à Émile Zola, 25 avril 1878 : « mon pédantesque roman » (Corr., V, p. 379). Il en va de cette aventure comme de la Révolution française : "Pour la juger impartialement, il faudrait avoir lu toutes les histoires, tous les mémoires, tous les journaux et toutes les pièces manuscrites, car de la moindre omission une erreur peut dépendre qui en amènera d'autres à l'infini » (op. cit., IV, p. 178).

19. Flaubert à Edma Roger des Genettes, 26 septembre 1874, Corr., IV, p. 866.

20. Perdus dans les philosophies de l'histoire, Bouvard et Pécuchet demandent le secours de Dumouchel. Celui-ci « avoua qu'il était maintenant dérouté en fait d'histoire. - " Elle change tous les jours. [...] C'est à souhaiter qu'on ne fasse plus de découvertes, et même l'Institut devrait établir une sorte de canon, prescrivant ce qu'il faut croire!" " (Corr., IV, p. 181). Hegel, que ses héros lisent sans y rien comprendre, l'avait bien dit: tout le savoir est en mouvement et l'Encyclopédie, même ironique comme celle de Flaubert, ne peut être que le mouvement même du Savoir.

21. Au bout de trois ans, Flaubert « les entrevoit maintenant [ses deux bonshommes] d'une façon plus vivante et moins artificielle » (à Edma Roger des Genettes, 15 février 1877, Corr., V, p. 186).

22. Jean-Paul Sartre, Les Mots, dans Les Mots et autres textes autobiographiques, Gallimard, coll. «Bibliothèque de la Pléiade », 2010, p. 137-138.

23. À propos de Pécuchet : «Son besoin de vérité devenait une soif ardente» (chap. VIII, p. 297). Et, plus haut, «Mais le goût de l'histoire leur était venu, le besoin de la vérité pour elle-même » (chap. IV, p. 178). L'ambition est celle de la philosophie; leur formation, leur culture et leurs moyens ne sont pas à la hauteur.

24. «Connaissez-vous Schopenhauer? - J'en lis deux livres. - Idéaliste et pessimiste, ou plutôt bouddhiste. Ça me va. » (à Edma Roger des Genettes, 13 juin 1879, Corr., V, p. 659). Sur Flaubert et Schopenhauer, voir l'article de Michel Brix, «Flaubert, Schopenhauer et le pessimisme », dans la Revue Flaubert $n^{\circ} 7$, Université de Rouen, 2007, Flaubert et la philosophie, sous la direction de Jacques Goetschel. 
INDEX

Mots-clés : savoir, Bouvard et Pécuchet, science, réalité

\section{AUTEUR}

PIERRE CAMPION

http://pierre.campion2.free.fr/ 